there could be no question of the ligature having dissolved away, as it was of strong silk, and the vessel was still pulseless below its seat ; moreover, pressure on the femoral at this spot did not affect the aneurysm, whereas pressure above the profunda did so at onco.

I now determined to try digital compression, and, on the readmission of the patient on January 26th, he was put upon a somewhat restricted diet for two days, being confined to bed, and smartly purged. The effect of this treatment in lowering the arterial tension was most marked, and to it, no doubt, some of the good result may be attributed. Then, on the 28th, having a number of most willing volunteers from among the students, I commenced digital pressure of the common femoral at 10.50 A.M. This was carried out with the same attention to details referred to in my former case, and, at 5.15 P.M., all pulsation in the aneurysm had ceased finally. Compression was still continued until 8 P.M., and then stopped. The temperature of the limb remained lower than the other for some time, but all discomfort and pain was soon gone. The patient returned home cured on February 5th. Since then, I have seen him several times; there has been no return of the aneurysm, which has shrunken up to small size. The pulsation in the tibials is still absent. The man is now engaged in his gardening work as before, without any pain or trouble from his former ailment.

\section{A CASE OF FACE-PRESENTATION.}

BY P. J. CREMEN, M.D.,

Physician to the Cork North Charitable Infirmary, and the Cork Union Hospital (Female Department).

ON June 19th, 1883, at 1 P.M., I was summoned to see Mrs. D. in labour. This was her third pregnancy. The membranes had burst at 6 A.M. on the same day. Slight labour-pains commenced at 8 A.M. Strong expulsive pains set in at 10, lasting until my amival. On each previous occasion, it was necessary to deliver her with the long forceps, in consequence of a narrowing of the conjugate diameter of the brim; and, when called on this occasion, I thought it was probably the same condition of things as before, namely, arrest of the head at the brim. However, on making an examination, I discovered that it was a face-presentation, and in the most unfavourable position-first mento-posterior. The presenting part had not yet entered the brim, although strong expulsive pains had lasted for the space of three hours. I decided, if possible, to change it into a vertex-presentation, as offering the best chance, in my opinion, both to mother and child.

The os uteri and soft parts were well dilated and cool. After passing the catheter, and emptying the rectum by enema, in the interval between the pains, I passed two fingers of my right hand over the vertex as far as they could reach, and applied the left hand externally over the hollow of the occiput as it lay in the left inguinal region. I felt I had complete control over the head, using gentle pressure externally with the left hand, aided by the two fingers of the right on the vertex internally. This served a double purpose; first, that of a tractor, and second as a director, preventing any contusion of soft parts. I was thus enabled to push down the vertex with very little difficulty into the right oblique diameter of the pelvis, thus changing the most unfavourable facial position into the most favourable cranial one.

I then left the case to nature, so that the head might mould itself into the cavity of the brim, and thus enable the woman to be safely delivered with the forceps, as, from previous knowledge of the case, I was satisfied that delivery would not be effected naturally. In two hours, the head fully occupied the brim; and, as a caput sucsedaneum was rapidly.forming, I applied the forceps, and had the satisfaction of delivering a healthy living male child.

REMARKS. - The treatment usually recommended in face-presentations in most of the modern works on obstetrics is to leave them to nature, although the mortality is stated by Playfair and others to be one in ten of the children when rotation takes place, and all lost when it does not take place. Why then leave such a case to nature if you see it before it enters the brim? Does it not, under the most favourable circumstances, involve a tedious labour and its consequences to the mother, and a certain risk to the child? I am certainly inclined to agree with Hodge, so far at least as mento-posterior positions are concerned, when he argues that they should be all changed into vertexpresentations, and for the valid reason of diminishing infantile mortality, and sparing the mother the effects of a tedious labour. One of the most recent authorities states it is more difficult and painful to the mother to bring down the vertex than the head, and prefers podalic version.

The slight operation of substituting the vertex for the face above the brim, in my limited experience, bears no comparison to podalic version, for in the latter, as we are well aware, the matermal mortality is one in fourteen, that of children one in three. In the case related it would have indeed been difficult, and not unattended with danger, to perform podalic version, when the waters had escaped for seven hours, and when the uterus was contracting powerfully. On the other hand, the operation of bringing down the vertex was accomplished with the greatest ease, and with little or no pain to the mother. This, however, I think it right to state, was mainly due to the external pressure.

This plan is one which, I feel confident, will prove successful in the hands of any accoucheur of ordinary experience, in cases where the face is yet above the brim.

\section{A NEW EFFERVESCING CHALYBEATE SPRING AT STRATHPEFFER SPA, N.B.}

\section{By D. MANSON, M.D.}

I wish to bring to the notice of the medical profession a new effervescing chalybeate at this Spa. For centuries the spring has been well known in the Scottish Highlands as the "Iron Well." It rises at the foot of Ben Wyvis, about three miles from the Spa, and was brought in iron pipes to the Pump-room a few years ago. Up to the present time, the water had stood, on its way to the Pump-room, first in a large deep open well where it rises, and then at the Spa-buildings in an open filter, allowing of the partial precipitation of the iron at each place from atmospheric oxidation, and the escape of carbonic acid gas, the iron existing in the spring as a carbonate of the protoxide. The filter having now been done away with, and the well emptied down to the effluent pipe at its bottom, the result is the conservation of the iron and of a very large amount of free carbonic acid gas in the water, with the delivery at the now permanently open taps in the Pump-room of an opalescent highly effervescent chalybeate, which, when received in the drinking-glass, gradually clears up like champagne. Like the most used and the most successful iron waters, it contaifs the essentially small quantity of iron (in its case, $\frac{1}{4}$ grain of the carbonate to the 10 ounces); and as the supply of the water is abundant (900 gallons per day), there will be sufficient for carbonated chalybeate baths, in addition to the present sulphur-bath establishment of the Spa. Besides, therefore, Strathpeffer possessing sulphursprings, among the strongest of their class in Europe, as well as recently established facilities for the inhalation of sulphuretted hydrogen-vapour, it will now have the advantage of a first-class acidulous chalybeate, to enhance its attractions as a Highland health-resort.

\section{CLINICAL MEMORANDA.}

\section{DIAGNOSTIC VALUE OF SPUTUM.}

THe very interesting contribution by Dr. Gabbett in the Journal of April 26th, on the diagnostic value of sputum stained by Koch's method, reminds me of a case which occurred here in March last, occurring in a patient whom I saw in consultation with my partner, Mr. Gowing. The patient was a young lady, aged 16, for whom Mr. Gowing had been consulted first on February 19th, for what was said to be a slight cough. He informed me that he considered the case to be suspiciously phthisical, although there was not sufficient evidence to justify at first a positive diagnosis. I saw her and examined her carefully, and, with the exception of a limited dulness over the upper border of the left scapula, and some crepitation in the same region, and a temperature varying from $100^{\circ}$ to $102^{\circ}$, which had persisted for some days, material evidence of phthisis was conspicuous by its absence. The girl was plump, and apparently well nourished, with good appetite until within a day or two of my visit; bowels and catamenia regular. She slept well and coughed very little; and had had then no expectoration. In view of the temperature and dulness, I concurred in the diagnosis, and suggested an examination of any sputum that could be obtained. As soon as some could be procured, I prepared and stained it according to Dr. Heneage Gibbes' method, when swarms of bacilli were at once apparent. Thereupon, a most unfavourable prognosis was given, and the patient was removed to her home on the first suitable day. She died of acute phthisis on April 21st. After her removal, the right lung was very quickly invaded.

In mentioning this case, I do not, for obvious reasons, wish to draw any positive conclusions; such can only be justified by a far larger amount of material than anyone can encounter in private practice. Nevertheless, we assumed for once the truth of the dictum "No bacilli (i.e., the bacilli which alone stain by the method we employed) 